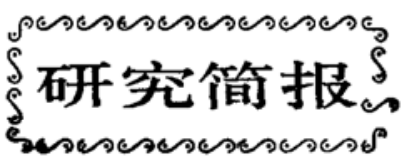

\title{
$A F-$ 代数及其维数群的分类
}

\author{
黄 昭 波 \\ (星且大学数学研究所, 上海 200433)
}

\section{关链词 $\boldsymbol{A} F$-代数、维数群、泛函、阿基米德元}

\section{一、引 言}

分类是算子代数的一个重要研究方向, Von Neumann 代数的分类已有许多工作. 近二 十年来, 随着算子 $K$-理论的发展, $C^{*}$-代数的分类问题引起了人们的注意, 但由于它的复杂 性, 没有取得大的进展. Cuntz 和 Pedersen 仿照 Von Neumann 代数的情形引进了有限、 半有限和纯无限的 $C^{*}-$ 代数的概念 ${ }^{[1]}$. 本文研究 $A F-$ 代数的这种分类. 我们在维数群中引进 了一些新的概念, 并利用这些概念完全刻划了 $A F-$ 代数的分类.

\section{二、 $\boldsymbol{A F}$-代数的分类和维数群的泛函}

定义 1 设 $A$ 是 $A F$-代数, $\left(G(A), G(A)_{+}, \Gamma(A)\right)$ 表示 $A$ 的标度维数群 ${ }^{[2]}$. 如果 $f$ 是 $G(A)$ 上的泛函 ${ }^{[2]}$, 并且满足:

$$
\sup \{f(a) \mid a \in \Gamma(A)\}<+\infty,
$$

则称 $f$ 是 $G(A)$ 的有界泛函.

定理 1 设 $A$ 是 $A F-$ 代数, 那末 $A$ 是有限的充要条件是 $G(A)$ 有忠实的有界泛函.

定义 2 设 $A$ 是 $A F$-代数, $\left(G(A), G(A)_{+}, \Gamma(A)\right)$ 是其标度维数群, $\varphi$ 是从 $G(A)_{+}$ 到 $R_{+} \cup\{+\infty\}$ 的映照, 其中 $R_{+}$表示非负实数的全体. 如果 $\varphi(a+b)-\varphi(a)+\varphi(b)$, $\forall a, b \in G(A)_{+}$, 则称 $\Phi$ 是 $G(A)$ 的广义泛函; 如果对于 $\forall b \in G(A)_{+} \backslash\{0\}$, 存在 $a, 0<a \leqslant$ $b$, 使得 $\varphi(a)<+\infty$, 则称 $\varphi$ 是半有限的广义泛函.

定理 2 设 $A$ 是 $A F$ - 代数, 那末

1) $A$ 是半有限的充要条件是 $G(A)$ 有半有限的忠实的广义泛函;

2) $A$ 是纯无限的充要条件是 $G(A)$ 不存在非平凡的忠实的广义泛函. ( $G(A)$ 的泛函 $\varphi(a)=0 \forall a \in G(A)_{+}$, 或 $\varphi(0)-0, \varphi(a)=+\infty, \forall a \in G(A)_{+} \backslash\{0\}$, 称为平凡的广义 泛函).

以上两个定理给出了 $A F$-代数的类型与其维数群上泛函之间的关系，其证明的关键在于 研究 $A F$-代数的迹和它的维数群上泛函之间的关系. 由此, 我们就把 $A F-$ 代数的分类问题转 化成 $G(A)$ 上的泛函的存在性问题.

1991-10-22 收稿, 1992-05-20 收修改稿 


\section{三、AF-代数类型的刻划}

首先考虑有限的情形,为此给出:

定义 3 设 $\left(G, G_{+}, \Gamma\right)$ 是一个标度维数群, $N$ 是自然数集.

1) 如果 $a \in \Gamma$, 并且对于 $\forall n \in N$, 都有 $n a \in \Gamma$, 则称 $a$ 是 $\Gamma$ 的稳定元; 否则, 即存在 $n_{0} \in N$, 使得 $n_{0} a \in \Gamma$, 则称 $a$ 为 $\Gamma$ 的有限元;

2) 如果 $\Gamma$ 中的任何非零元都是有限元, 则称 $\Gamma$ 是有限的, 这时称维数群 $G$ 具有有限标度 $\boldsymbol{\Gamma}$.

定理 3 设 $\left(G, G_{+}, \Gamma\right)$ 是标度维数群,那末下面论断等价:

1) $\Gamma$ 是有限标度;

2) 对于 $\forall a \in \Gamma \backslash\{0\}$, 都存在 $G$ 的有界泛函 $\varphi$, 使得 $\varphi(a)>0$;

3) $G$ 有忠实的有界泛函.

由此, 我们可以给出有限 $A F-$ 代数的特征.

定理 4 如果 $A$ 是 $A F$-代数, 那末 $A$ 是有限的充要条件是其维数群 $\left(G(A), G(A)_{+}\right.$. $\Gamma(A))$ 的标度 $\Gamma(A)$ 是有限的.

这个定理推广了 Blackadar ${ }^{[3]}$, Goodearl 和 Handleman ${ }^{[4]}$ 以及 Cuntz 和 Pedersen ${ }^{[1]}$ 中 的相应结论.

为了刻划无限的 $A F$ 代数, 我们给出下面的

定义 4 设 $\left(G, G_{+}\right)$是维数群.

1) 如果 $b \in G_{+}$, 并且对于 $G_{+}$中的任意的非零元 $a$, 都存在自然数 $n_{0}$, 使得 $n_{0} a \leqslant b$ 不 成立, 则称 $b$ 是 $G$ 的阿基米德元;

2) 如果 $b \in G_{+} \backslash\{0\}$, 对于 $\forall a \in G_{+} \backslash\{0\}, a \leqslant b$, 都存在 $c \in G_{+} \backslash\{0\}$, 使 得 $n c \leqslant a$. $\forall n \in N$ 都成立, 则称 $b$ 是纯无限的;

3）如果 $G_{+}$中的任何元都是阿基米德的,则称 $G$ 是阿基米德的; 如果 $G_{+}$中不存在非零的 阿基米德元, 则称 $G$ 是纯无限的;

4) 如果 $a \in G_{+} \backslash\{0\}$, 并且存在阿基米德元 $b \in G_{+} \backslash\{0\}$, 使得 $b \leqslant a$, 则称 $a$ 是 $G$ 的半 有限元; 如果 $G_{+}$中的每个非零元都是半有限的, 则称 $G$ 是半有限的.

注 阿基米德元的概念是本文首次从 Riesz 空间以中移植到维数群中的. 另外, 定义 3 中的有限标度的概念也是本文首次引进的.

定理 5 如果 $A$ 是 $A F$-代数,则下面论断等价:

1) $A$ 是半有限的;

2) $G(A)$ 具有忠实的半有限的广义泛函;

3) $G(A)$ 含有阿基米德的本质理想 ${ }^{\omega}$;

4) $G(A)$ 含有有限标度的本质理想.

定理 6 如果 $A$ 是 $A F-$ 代数, 那末 $A$ 是半有限(或纯无限)的充要条件是 $G(A)$ 为半有 限(或纯无限)的维数群.

\section{四、应用}

利用纯无限 $A F-$-代数的维数群的特征,我们可以给出纯无限的 $A F-$ 代数(即文献 [1]中的 
III 型)的一些例子.

我们称 $C^{*}-$ 代数 $C$ 是 $C^{*}-$ 代数 $A 、 B$ 的 $C^{*}-$ 扩张, 如果

$$
0 \rightarrow A \rightarrow B \rightarrow C \rightarrow 0
$$

是 $C^{*}$-代数的短正合列. 对于 $A F$-代数的 $C^{*}$-扩张, 利用上述结果以及算子 $K$-理 论中的 “six-term” 正合列 ${ }^{[6]}$, 我们证明了

定理 7 如果 $A 、 B$ 都是有限的(半有限的、纯无限的) $A F$-代数, 那末 $A, B$ 的 任 何 $C^{*}$-扩张也都是有限的(半有限的、纯无限的) $A F-$ 代数.

\section{参考文献}

[1] Cuntz, J., Pedersen, G. K., J. Funct. Anal., 33(1979), 135-164.

[2] Effros, E., Dimensions and C*-algebras, CBMS Regional Conference Series in Math. AMS, 46(1981), $23,43$.

[ 3 ] Blackadar, B., J. Funct. Anal., 38(1980), 156-168.

[4] Goodearl, K., Handleman, D., J. Keine Angew. Math., 332(1982), 1-98.

[5] Luxemburg, W.. Zaanen, A., Riesz Spaces, Vol.1, North-Holland Pub. Co., Amsterdam, London, $1971,112$.

[6] Blackadar, B., K-Theory for Operator Thcory, MRSI, Springer-Verlag, 1986, 77. 